\title{
Abnormal muscle and skin mitochondria in family with myoclonus, ataxia, and deafness (May and White syndrome)
}

\author{
J Vaamonde, J Muruzabal, T Tuñón, N Perez, J Artieda, M Rodriguez, J A Obeso
}

\begin{abstract}
A mother and two of her daughters had deafness and cortical reflex myoclonus; the mother also had mild truncal ataxia. Muscle and skin biopsy specimens revealed abundant ragged-red fibres and abnormal mitochondria. The son of one of the daughters had sensorineural deafness. Three other grandchildren were asymptomatic. The two daughters also had diabetes mellitus, hypertension and cardiomyopathy. Another daughter died of renal failure. The mother lost her hearing in her 70s, one daughter in her 30 s, and the other daughter and the grandson in their 20s. The mother has had transient episodes (24-48 hours) of temporal disorientation, severe action myoclonus, and ataxia for about eight years. This is the first reported family with inherited deafness, myoclonus, and ataxia with mitochondrial pathology.
\end{abstract}

In 1965 May and White described a family with inherited deafness, myoclonus, and ataxia. ${ }^{1}$ Three other similar families have been subsequently reported. ${ }^{2-4}$ Necropsies in the family reported by Baraitser et ll $^{2}$ showed loss of cells in the dentate nuclei, decreased cerebellar white matter, and minor alterations of the gracile tracts in the spinal cord. In the two other families conventional and electromicroscopic muscle biopsy studies were reported as normal. ${ }^{34} \mathrm{~A}$ few other families with myoclonus, including photically evoked myoclonus, ataxia, and hearing loss, diabetes, and nephropathy ${ }^{56}$ or lipoma, ${ }^{7}$ in different combinations have been described without any specific pathological finding. Recently, Berkovic et $a l^{\beta}$ called attention to the clinical overlap between such families and patients with myoclonic epilepsy with ragged-red fibres (MERRF). We describe a family with myoclonus, ataxia, deafness, diabetes, and hypertension in whom skin and muscle biopsy specimens showed evidence of mitochondrial disease.

\section{Patients and methods}

Figure 1 summarises the family pedigree and main clinical features. The proband was an 80 year old woman with seven siblings (three females and four males) and three daughters. Precise clinical information about the proband's parents is lacking. They apparently died at a late age with no major health problem. Two daughters were affected, and another died at the age of 23 years of renal failure. A grandson suffered from hearing loss. Two siblings of the proband died at an early age with no medical diagnosis, and two died in accidents. Two brothers and one sister are still alive. One 78 year old brother is deaf; the two other siblings are healthy at the age of 77 (brother) and 62 (sister). Children of these family members are considered normal medically but have not been examined by us.

\section{Case reports}

Index case II.5

Patient II.5 was an 80 year old woman with an eight year history of episodic jerking of the limbs accompanied by confusion and disorientation. Each episode lasted for three to five days with variable but usually monthly presentation. There was no recognised precipitant factor. She had a history of progressive hearing loss for the last 15 years. There was no history of hypertension, cardiopathy, diabetes, renal failure, or drug use. Examination showed normal general physical features. There was bilateral hearing loss. Her gait was slightly unsteady. Romberg's sign was negative. Touching and pinpricking the hands or feet produced reflex muscle jerking with focal distribution. Ocular pursuit movements were sluggish and broken in microsaccades, but there was no ophthalmoplegia. Examination was otherwise normal. During the acute episodes there were continuous myoclonic
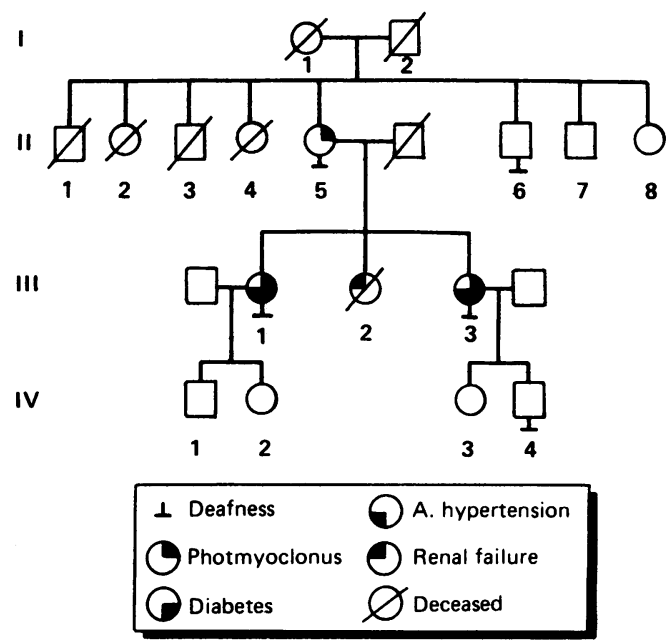

Figure 1 Family pedigree showing members with myoclonus, ataxia, hearing loss, diabetes, and hypertension. 
Figure 2 CT brain scan (unenhanced) from patient III.1 shows clear atrophy of brainstem and cerebellum but minimal supratentorial involvement.

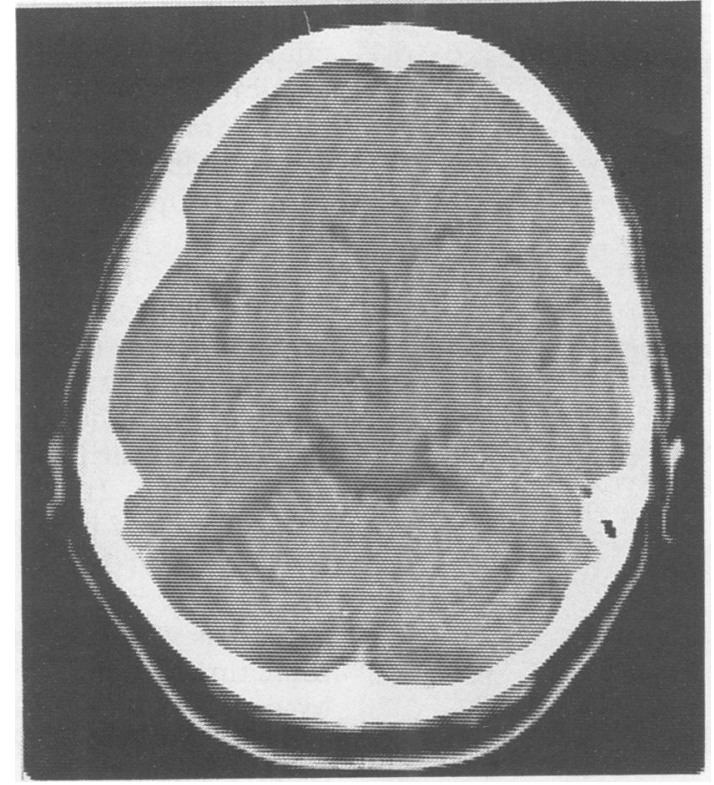

Assessment of auditory function

The study of auditory function established the existence of asymmetrical sensorineural hearing loss in four patients (II.5, III.1, III.3, and IV.4). The three other grandchildren did not wish to be examined. Hearing loss was moderate in patients II.5 and IV.4, (pure tone average $40-60 \mathrm{~dB}$ ) and severe in patients III.1 and III.3 (pure tone average 60-90 dB). Brainstem auditory evoked potentials to 80 and 100 dB clicks had normal onset and inter-peak latencies but were decreased in amplitude in all four patients. In the test known as "sound intensity shift index" (SISI) patients II.5, III.1, and III.3 could discriminate minimal threshold variations in sound intensity. This response of "positive recruitment" is typical of cochlear pathology. There was no tone decay. Stapedius reflex thresholds were normal at all frequencies, and the speech audiograms were altered as predicted by the degree of tone threshold alterations. Overall, the auditory tests strongly indicated an asymmetrical cochlear origin for the hearing loss of the four affected family members. Vestibular function was assessed by independent stimulation of the ears with water at $30^{\circ} \mathrm{C}$ and $44^{\circ} \mathrm{C}$ (Fitzgerald and Hallpike caloric test) and recording of the slow phase of the oculovestibular reflex. This but disoriented temporally and spatially. All blood tests were normal. CT brain scan showed generalised cortico-subcortical atrophy predominating in the posterior fossa.

\section{Case III.1}

This 47 year old woman, daughter of case II.5, had no relevant medical history until the age of 22 when she developed bilateral hearing loss. At 34 years a diagnosis of hypertrophic cardiomyopathy and hypertension was made, and a few years later she was started on insulin for diabetes. Myoclonic jerks in the limbs developed at 36 years, and treatment with sodium valproate ( $1200 \mathrm{mg}$ daily) was initiated achieving adequate control. Neurological examination in 1988 was entirely normal except for hearing loss. Blood tests revealed a glucose concentration of $1580 \mathrm{mg} / 1$ but were otherwise normal. Cardiological assessment confirmed the diagnosis of hypertrophic cardiomyopathy. A CT brain scan showed clear cerebellar and brainstem atrophy with minimal involvement of supratentorial structures (fig 2).

\section{Case III.3}

This 44 year old woman, another daughter of case II.5, had normal development. Hearing loss began at the age of 13 years. At 40 years an EEG showed photosensitivity. She was also diagnosed at that time as having hypertension, diabetes, and hypertrophic cardiomyopathy. In 1989 neurological examination was normal. The above diagnoses were confirmed. A CT brain scan showed moderate cortico-subcortical atrophy. All blood tests were normal.

\section{Case IV.4}

This 14 year old boy is the son of case III.3. He is entirely normal except for bilateral hearing loss.
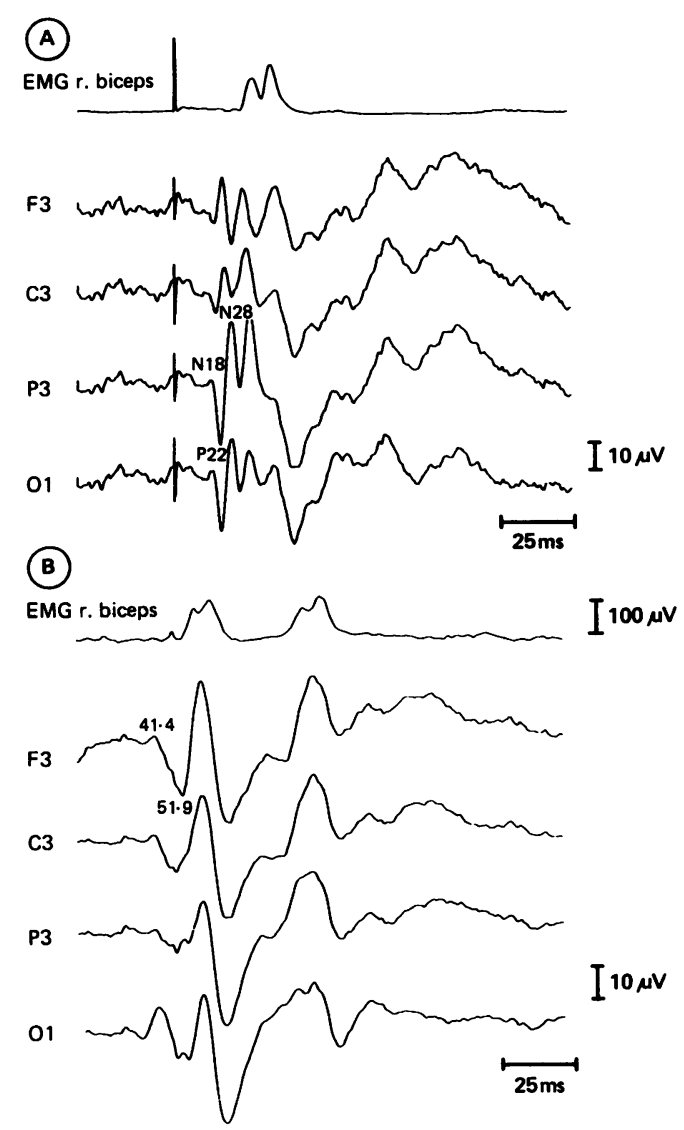

Figure $3 A$ Electromyographic (EMG) activity in right biceps (top channel) and SEPs (3 bottom channels) recorded from left frontal (F3), rolandic (C3), parietal $(P 3)$, and occipital (01) regions after right median nerve stimulation. Giant SEP predominantly over $P 3$ is stimulation. Giant SEP predominantly over $P 3$ is in biceps. B EMG and cortical electrodes as in A. Flash stimulation at $1 \mathrm{~Hz}$ induced normal visual evoked potential in occipital cortex (01) followed by giant frontal response with latency to onset of $41.4 \mathrm{~ms}$. Latter wave preceded myoclonic jerk by $10.5 \mathrm{~ms}$. 

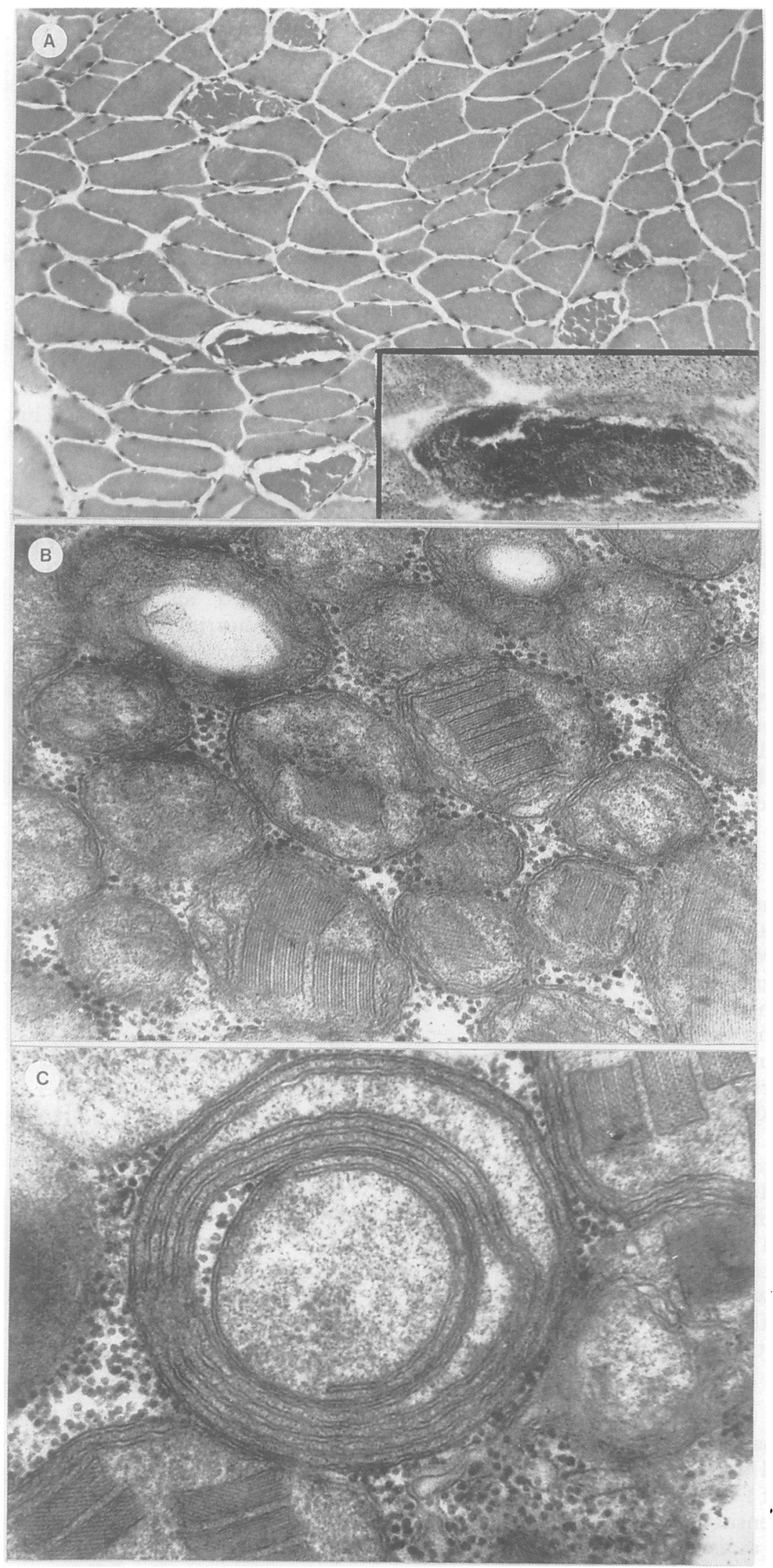

Figure $4 A$ Section of muscle (from patient DM) stained with haematoxylin-eosin $(\times 125)$ showing several ragged-red fibres with intense positive reaction by succinic dehydrogenase stain (insert magnification $\times 500$ ). B and $C$ Electron micrographs showing mitochondrial paracrystalline inclusions $(B, \times 7000)$ and whorled membranes $(C, \times 11000)$.
Microsaccadic oscillations (square waves) were not recorded in central gaze position. Central fixation was abnormal due to hypometric saccades. Smooth pursuit (sinusoidal pendulum, $40 \mathrm{~Hz}, 40 \% \mathrm{~s}$ ) was decomposed in microsaccades. Optokinetic nystagmus was normal.

Electrophysiological assessment of myoclonus

Somatosensory evoked potentials (SEPs) after median nerve stimulation at the wrist were recorded from the scalp $(\mathrm{C} 3, \mathrm{C} 4, \mathrm{~F} 3, \mathrm{~F} 4)$ at the same time as the rectified EMG response from the biceps. ${ }^{910}$ In a different session, visual cortical evoked potentials to flash stimulation $(1-15 \mathrm{~Hz})$ and the rectified EMG activity in the biceps were also recorded. In each test 128 potentials were averaged and repeated at least three times. Photosensitivity was also assessed by conventional EEG recording. Patient II.5 had giant SEPs (figure 3A) associated with a reflex EMG discharge. The amplitude of the N20-P22 and P22-N33 waves after right median stimulation was $13.6 \mu \mathrm{v}$ and $28.4 \mu \mathrm{v}$ respectively. The latency of the double EMG discharge in the biceps was $33.3 \mathrm{~ms}$ and $44.4 \mathrm{~ms}$. Flash stimulation at $1 \mathrm{~Hz}$ produced a generalised myoclonic jerk with a latency of $55.2 \mathrm{~ms}$ to the biceps. A very large $(38.2 \mu \mathrm{V})$ occipito-frontal potential preceded the myoclonic discharge by $10.5 \mathrm{~ms}$ (figure $3 \mathrm{~B}$ ). Patients III.1 and III.3 had SEPs with an amplitude (P22-N33) of 9.3 and 14.4 $\mu \mathrm{V}$ over the left hemisphere and 9.7 and $14.2 \mu \mathrm{V}$ over the right hemisphere. In both patients a reflex EMG discharge was recorded in the arms with a latency of $37.5 \mathrm{~ms}$ in finger extensors (patient III.1) and $42 \mathrm{~ms}$ in finger flexors (patient III.3). In both patients flash stimulation (15$18 \mathrm{~Hz}$ ) induced generalised myoclonic jerks accompanied by a giant cortical potential with frontal predominance, similar to the one shown in figure 3B. Photosensitivity was not present in patient IV.4. Nerve conduction and EMG study in the three adult patients did not disclose peripheral nerve or muscle abnormalities.

\section{Muscle and skin biopsies}

Quadriceps biopsies were performed in the three adult (II.5, III.3, III.1) patients. Tissue was fixed with methyl-butane precooled in liquid nitrogen. Frozen sections were stained with haematoxylin and eosin, succinic dehydrogenase (SDH), nicotinamide adenine dehydrogenase (NADH), PAS, and the modified Gomori trichrome stain. Similar findings were observed in all three patients. Numerous ragged-red fibres and atrophic fibres were seen at low power magnification (figure 4A). These fibres showed excessive oxidative enzyme activity with SDH and NADH. Ultrastructural study showed abnormal mitochondria with typical paracrystalline inclusions (figure 4B). Mitochondria were enlarged and had whorled membranes (figure 4C). Lipofuscin was abundantly accumulated in many fibres. Similar mitochondrial abnormalities and lipofuscin accumulation were observed in the skin. 


\section{Discussion}

The clinical features of this family fall between those of the families described by May and White ${ }^{1}$ and Hermann et al. ${ }^{5}$ May and White's index case presented with deafness at the age of 4 years and at 14 years developed ataxia, severe action myoclonus, dysarthria, and, occasionally, generalised seizures. Ataxia and myoclonus caused great disability, but there was no dementia. The pattern of inheritance was thought to be autosomal dominant, but maternal transmission was also possible. Hermann et $a l^{5}$ described a family with photic myoclonus, grand-mal seizures, deafness, nephropathy, and diabetes mellitus in different combinations in three generations. Their proband's illness started in adult life and evolved progressively into a dementing state accompanied by severe myoclonic jerking. At necropsy the brain showed intense neuronal degeneration in both the cerebral and cerebellar cortex and moderate neuronal loss in the dentate nuclei and inferior olive. PASpositive lipid material was present in the remaining neurons in both nuclei. No evidence of spinal cord or peripheral nerve damage was found. The kidney showed nonspecific changes, which could be secondary to the diabetes. Another family with similar clinical findings and neuropathy, a progressive clinical evolution, and maternal inheritance was reported by Baraitser et al. ${ }^{2}$ Disease onset was in middle age. Necropsy of one patient revealed dentate nuclei atrophy and reduced white matter in the cerebellar hemispheres. Minor gliosis of the inferior olives and pallor of the myelin stain in the gracile tract was also noticed. Recently, Melo and Ferro ${ }^{4}$ described a family with ataxia, action myoclonus, and deafness associated with neuropathy and amyotrophy. The pattern of inheritance was autosomal dominant and incompatible with maternal transmission.

The clinical features of our family are undoubtedly similar to the previously reported families. The family tree suggests autosomal dominant or maternal inheritance. Deafness was the initial sign, as early as in adolescence in the later generations. Ataxia was a minor manifestation in the proband and so far absent in other family members. Action and spontaneous myoclonus has not been a major manifestation, but the three adults have clear cut electrophysiological features of cortical reflex myoclonus. ${ }^{910}$ Indeed photosensitivity has been a common finding in all families so far reported. ${ }^{1-7}$ We have not found evidence of nephropathy in the three adult family members studied in detail but one daughter died of renal failure and the presence of hypertension in the two other daughters (other causes excluded) is highly suggestive of renal dysfunction. Diabetes was present in two patients (III.1, III.3). Neither nephropathy nor diabetes have been associated with inherited ataxia and myoclonus, except in the family reported by Hermann et al. ${ }^{5}$ The association of hereditary renal failure and action myoclonus described in detail by Andermann et al seems to be an entirely different clinical condition. ${ }^{11}$
The family we describe is the first to be reported with abnormal muscle and skin mitochondria in May and White syndrome. The clinical evolution of our patients has been fairly benign. The proband has reached old age and enjoys a fairly normal life. The two sisters are now unlimited in activities of daily living. The wide spectrum of clinical features and evolution of the families so far reported is compatible with mitochondrial disease. ${ }^{12}$ The relation between inherited myoclonic syndromes and mitochondrial disorders is difficult to ascertain completely. Berkovic et $a l^{8}$ recently called attention to the high incidence of mitochondrial abnormalities in muscle and skin biopsy specimens from patients with progressive myoclonic epilepsy. Abnormal mitochondrial function, however, was a rare finding in two larger studies of patients with action myoclonus and ataxia but with minor epilepsy and no dementia as the main clinical features. ${ }^{1314}$ Mitochondrial disorders may be particularly common in patients with inherited myoclonus and ataxia accompanied by deafness or dementia or both.

Pathological findings at necropsy in two patients with May and White syndrome ${ }^{3}$ and from a few patients with MERRF ${ }^{1516}$ indicate multiple system degeneration (MSD). CT brain scans in our family, particularly in patient III.1, also showed the typical findings seen in patients with MSD. ${ }^{16}{ }^{17}$ Recently, a defect in the complex I respiratory chain has been described in Parkinson's disease. ${ }^{18}$ Similar changes are produced in substantia nigra neurons by MPTP administration in animals. ${ }^{19}$ Defective mitochondrial function may be the final mechanism leading to neuronal death secondary to environmental and endogenous (genetically determined) toxins.

We thank Professor A Harding (London) for reviewing the paper and Mrs M Mar López and Carol Elsden for helping in its preparation.

1 May DL, White HH. Familial myoclonus, cerebellar ataxia and deafness. Arch Neurol 1968;19:331-8.

2 Baraitser M, Gooddy W, Halliday AM, Harding AE, Rudge P, Scaravilli F. Autosomal dominant late onset cerebellar ataxia with myoclonus, peripheral neuropathy and sensorineural deafness: a clinicopathological report. $J$ Neurol Neurosurg Psychiatry 1984;47:21-5.

3 Chayasirisobhon S, Walters B. Familial syndrome of deafness, myoclonus and cerebellar ataxia. Neurology 1984; 34:78-9.

4 Melo TP, Ferro JM. Autosomal dominant cerebellar ataxia with deafness, myoclonus and amyotrophy. J Neurol with deafness, myoclonus and amy
Neurosurg Psychiatry 1989;52:1448-9.

5 Herrmann C, Agilar MJ, Sacks OW. Hereditary photomyoclonus associated with diabetes mellitus, deafness, nephropathy and cerebral dysfunction. Neurology 1964;14:212-21.

6 Schafer IA, Scriver CR, Efron ML. Familial hyperprolinemia, cerebral dysfunction and renal anomalies occurring in a family with hereditary nephropathy and deafness. N Engl J Med 1962;267:51-60.

7 Ekbom K. Hereditary ataxia, photomyoclonus, skeletal deformities and lipoma. Acta Neurol Scand 1975;51 393-404.

8 Berkovic F, Carpenter S, Evans A, et al. Myoclonus epilepsy and ragged-red fibres (MERRF). Brain 1989;112: $1231-60$.

9 Hallett $M$, Chadwick D, Marsden $C D$. Cortical reflex myoclonus. Neurology 1979;29:1 107-25.

10 Obeso JA, Rothwell JC, Marsden CD. The spectrum of cortical myoclonus. Brain 1986;108:193-224.

11 Andermann E, Andermann F, Carpenter S, et al. Action myoclonus-renal failure syndrome: a previously unrecogmyoclonus-renal failure syndrome: a previously unrecognephrology. Adv Neurol 1986;43:87-104. 
12 Petty RKH, Harding AE, Morgan Hughes JA. The clinical features of mitochondrial myopathy. Brain 1986;109: 915-38.

13 Tassinari CA, Michelicci R, Genton P, Pellisier JF, Roger J Dysinergia cerebellaris myoclonica (Ramsay Hun syndrome) a condition unrelated to mitochondria encephalomyopathies. J Neurol Neurosurg Psychiatry 1989;52:262-5.

14 Marsden CD, Harding AE, Obeso JA. Progressive myoclonic ataxia (the Ramsay Hunt syndrome). Arch Neurol 1990;47:1121-5.

15 Lombes A, Mendell JR, Nakase H, et al. Myoclonic epilepsy and ragged-red fibres with cytochromic oxidase deficiency: neuropathology, biochemistry and molecular genetics. Ann Neurol 1989;26:20-33.

16 Truong DD, Harding AE, Scaravilli F, Smith SJM, Morgan-Hughes JA, Marsden CD. Movement disorders in mitochondrial myopathies. A study of nine cases with two autopsy studies. Movement Disorders 1990;5:109-17. 17 Ramos R, Quintana F, Diez C, Lera C, Berciano J. CT findings in spinocerebellar degeneration. $A J N R$ 1987; 8:635-40.

18 Schapira AH, Cooper JM, Dexter D, Jenner P, Clark JB Marsden CD. Mitochondrial complex I deficiency in Parkinson's disease. Lancet 1989;i:1269.

19 Sayre LM. Biochemical mechanisms of action of the dopaminergic neurotoxin 1-methyl-4-phenyl- 1, 2, 3, 6 tetrahydropyridine (MPTP). Toxicol 1989;48:121-49. 